\title{
Urinary Interleukin-8 as a Biomarker for Steroid Resistance in Childhood Onset Nephrotic Syndrome
}

\author{
Heba Mostafa Ahmed', Osama Ezzat Botrous', Rasha Abd ElRazek Khattab², \\ Ahmed Mohammed Abdallah' \\ 1- Department of Pediatrics, Faculty of Medicine, Beni- Suef University, Egypt. \\ 2- Department of Clinical and Chemical Pathology, Faculty of Medicine, Beni- Suef University, Egypt.
}

\begin{abstract}
Introduction

Nephrotic syndrome (NS) is one of the commonest pediatric renal disorders. Minimal change disease (MCD) is the commonest cause of childhood NS followed by focal segmental glomerulosclerosis (FSGS). The majority of MCD children respond to corticosteroid therapy, but some are steroid-dependent or steroid-resistant, while the majority of patients with FSGS are steroid resistant. Recently, it has been proposed that changes in the cytokine and chemokine profile of NS patients may contribute to proteinuria and glomerular damage
\end{abstract}

\section{Aim of this study}

To investigate the relation between interleukin 8 (CXCL8/IL8) and the response to steroid therapy in pediatric nephrotic syndrome.

\section{Patients and methods}

Sixty-five patients with NS were included. They were divided into two groups according to response to steroids group 1: steroid sensitive NS (40 patients) group 2: steroid resistant NS (25 patients) and each group include two subgroups (during disease activity and during remission). Serum and urine CXCL8/IL8 levels were measured by quantitative sandwich enzyme immunoassay technique.

\section{Results}

During activity serum CXCL8/IL8 levels were significantly higher in SRNS [median, IOR = 293(11-296)] as compared to SSNS [114.3(5.8-296)] , p .04. Similarly, urinary CXCL8/IL8 levels were significantly higher in SRNS [187(61.8-210.3)] as compared to SSNS [40.6(24.4-80)] , p.04. During the remission, urinary CXCL8/IL8 levels were significantly higher in SRNS [ 38(21.8-57)] as compared to SSNS [2.4(.0-9.8)], p .03.

\section{Conclusions}

Urinary CXCL8/IL8 levels were significantly higher in SRNS than SSNS patients and can be a reliable marker for steroid resistance of childhood onset nephrotic syndrome

\section{Keywords}

Nephrotic syndrome; Interleukin-8; Steroid resistance; Proteinuria

\section{Correspondence Heba Mostafa Ahmed}

Department of Pediatrics, Faculty of Medicine, Beni- Suef University, Egypt.

E-mail address: heba_most@yahoo.com

\author{
geget : The Journal of the Egyptian Society of Pediatric Nephrology and Transplantation (ESPNT) \\ geget https://geget.journals.ekb.eg/ \\ Published by ESPNT http://espnt.net/ \\ Cohosted by Egyptian Knowledge Bank https://www.ekb.eg
}




\section{Introduction}

Nephrotic syndrome (NS) is one of the commonest pediatric renal disorders characterized by proteinuria, hypo- albuminemia and generalized edema [1]. The incidence of nephrotic syndrome is ranging between two to seven newly diagnosed cases per 100,000 children per year, and the prevalence is about 16 cases per 100,000 children [2]. Minimal change disease (MCD) is the commonest cause of idiopathic childhood onset nephrotic syndrome. It accounts for about $85 \%$ of cases followed by focal segmental glomerulosclerosis (FSGS), in almost $10 \%$ of cases [3]. The majority of MCD children who present with nephrotic syndrome respond to corticosteroid therapy, but some are steroid-dependent or steroid-resistant, while the majority of patients with FSGS are steroid resistant with a high possibility to progress to end-stage renal disease (ESRD) [4]. It has been proposed that changes in the cytokine and chemokine profile of idiopathic nephrotic syndrome patients may contribute to proteinuria and glomerular damage $[5,6]$. Cytokines are a group of proteins produced by several kinds of cells that function as soluble mediators with intercellular signaling functions [7]. Interleukin-8 (CXCL8/IL8) is a chemoattractant cytokine produced by a variety of tissue and blood cells [8]. IL-8, mainly from lipopolysaccharide activated monocytes, antigen or phytohaemagglutinin activated $\mathrm{T}$ cells and resident kidney cells, serves as an important mediator of inflammatory diseases, causes accumulation and activation of inflammatory cellsin kidney and aggravates renal pathological lesion [9]. Moreover, by affecting the metabolism of sulfide in glomerular basement membrane, IL-8 alters its anionic charge barrier and induces proteinuria [10]. On the other hand some studies had linked between urinary excretion of CXCL8/IL8 and the pathogenesis of proteinuria in INS $[6,11]$. The aim of this study was to investigate the relation between CXCL8/IL8 and the response to steroid therapy.

\section{Patients and methods}

Sixty-five patients with idiopathic nephrotic syndrome were included in the study between March 2015 and October 2018. At the first presentation, all patients received oral prednisone at dose of $2 \mathrm{mg} / \mathrm{kg} /$ day for $6-8$ weeks. Patients with steroid resistance underwent renal biopsy and an immunosuppressive was added to induce remission. The patients were divided into 2 groups according to response to steroid therapy; group 1: steroid sensitive NS (SSNS) 40 patients and group 2: steroid resistant NS (SRNS) 25 patients. Each group was studied during proteinurea (a) and during remission (b) ( groups 1a, 1b, $2 \mathrm{a}, 2 \mathrm{~b})$ The SRNS group were divided into two subgroups according to renal pathology; group $2 \mathrm{~F}$ (FSGS, 16 patients) and group 2M (MCD, 8 patients). The exclusion criteria were: patients with partial remission, congenital nephrotic syndrome, 2ry nephrotic syndrome, diminished estimated glomerular filtration rate $\left(<60 \mathrm{ml} / \mathrm{min} .1 .73 \mathrm{~m}^{2}\right)$, any acute infectious (high CRP levels) or allergic disease at the moment and four weeks before blood and urine sampling. All children included in the study were subjected to a written consent from their parents, full history taking laying stress on, age of onset of the disease and medical treatment. Complete clinical examination including body weight, height, body mass index (BMI), blood pressure, and edema. Laboratory investigations including blood urea, serum creatinine, albumin, cholesterol, urinary protein/creatinine ratio (uPCR), serum CXCL8/IL8 and urinary (CXCL8/IL8) / creatinine ratio. Our local institutional ethics committee approved the study.

Study definitions:

$\circ$ Steroid-sensitive NS: complete remission of INS obtained after a 6-8-wk course of corticosteroids.

- SRNS: no remission or only partial remission obtained after an 8-wk course of corticosteroids.

- Complete remission: protein / creatinine less than 0.2 $\mathrm{mg} / \mathrm{mg}$ creat and serum albumin more than $3.5 \mathrm{~g} / \mathrm{dl}$

- Partial remission: protein / creatinine more than 0.2 $\mathrm{mg} / \mathrm{mg}$ creat and serum albumin more than $2.5 \mathrm{~g} / \mathrm{dl}$

- Disease activity : protein / creatinine more than 2 $\mathrm{mg} / \mathrm{mg}$ creat and serum albumin less than $2.5 \mathrm{~g} / \mathrm{dl}$ [12].

Sample collection: Both serum and urine samples were collected at the presentation during proteinurea before starting steroid therapy and once again, when complete remission is obtained and maintained for at least two months.

Urine samples: The samples were taken as early morning samples being voided directly into a sterile container. Samples were divided into parts; one part sent for measurement of urinary proteins and creatinine. the other part was centrifuged for 20-min at the speed of 2000-3000 r.p.m. to remove supernatant and stored at $-80^{\circ} \mathrm{C}$ till time of analysis for quantitative determination of urinary CXCL8/IL8 applying sandwich Enzyme linked Immune Sorbent Assay technique (ELISA) using CXCL8/IL8 ELISA Kit, Thermo Fisher Scientific, USA ).

Serum samples: Venous blood sample of $4 \mathrm{ml}$ was withdrawn from each child. Samples were allowed to clot for two hours at room temperature before centrifugation for 15 minutes at $1000 x g$. Serum was separated and samples were divided; one .part sent for chemical analysis and the 2nd part was stored at $-80 \mathrm{C}$ until the end of the study for measurement of serum CXCL8/IL8 levels by quantitative sandwich enzyme immunoassay technique using CXCL8/IL8 ELISA Kit, Thermo Fisher Scientific, USA).

\section{Statistical methods}

Statistical analysis was done using statistical package for social sciences (SPSS), computer software (version22), IBM software, USA. Data were described in the form of median (IQR) for quantitative data, and frequency and proportions for qualitative data. A $p$ value $<0.05$ was considered statistically significant. Differences were analyzed between the groups by One-way Anova test. Associations were analyzed by logistic regression tests. The receiver operating characteristics (ROC) curve analysis was performed to identify the optimal cutoff value for urinary CXCL8/IL8/ creatinine level above which steroid resistance is expected. 


\section{Results}

There was no significant difference between the four groups as regarding age, age at disease onset and anthropometric measures. Blood urea and urinary CXCL8/IL8/creat were significantly higher in SRNS during activity as compared to the other groups ( $\mathrm{p}=.02$ and $>.001$ respectively. (table 1 ). In comparing the SSNS and SRNS during activity serum CXCL8/IL8 levels were significantly higher in SRNS (median, IOR $=293(11-296)$ as compared to SSNS (median, IQR $=114.3$ (5.8-296), $\mathrm{p}$ value $=.04$. Similarly, urinary CXCL8/IL8 / creat levels were significantly higher in SRNS (median , IOR = 187(61.8-210.3) as compared to SSNS (median, IQR $=40.6(24.4-80), \mathrm{p}$ value $=.04$ while there was no significant difference between the two groups as regarding serum albumin and PCR (table2). On comparing the SSNS and SRNS groups during the remission; urinary CXCL8/IL8 / creat levels were significantly higher in SRNS (median, $\mathrm{IOR}=38(21.8-57)$ as compared to SSNS (median, IQR $=2.4(.0-9.8), \mathrm{p}$ value $=.03$ while there was no significant difference between the two groups as regarding serum albumin, PCR and serum CXCL8/IL8 levels (table 3). All patients with SRNS were on immunesuppressants $(68 \%$ on cyclosporine and $32 \%$ on MMF; eight patients, seven of them had MCD and 1 patient with MesPGN) ). The renal pathology in the SRNS groups were (16 patients with FSGS, 8 patients with MCD and 1 patient had mesangial proliferation glomerulonephritis (MesPGN). There was no significant difference between FSGS and MCD as regarding serum and urinary IL8 levels during both active disease and remission ( $\mathrm{p}>.05)$. Binary logistic regression test showed that urinary IL8 levels can significantly predict the response to steroid therapy. $\left(\chi^{2}=\right.$ (27.7x 65) .p <.001) (Table 4). The area under the ROC curve of urine CXCL8/IL8 levels for the diagnosis of SRNS during active disease was 0.94 (95\% confidence interval, 0.81 to 0.99$)$. The analysis rendered an optimal cutoff value of $>35.3 \mathrm{pg} / \mathrm{mg}$ creatinine corresponding to 92 , $9 \%$ sensitivity and $84.6 \%$ specificity (Figure 1).

Table 1: Demographic and laboratory data of the study groups

\begin{tabular}{|c|c|c|c|c|c|}
\hline & $\begin{array}{l}\text { SSNS in } \\
\text { remission }\end{array}$ & $\begin{array}{l}\text { SSNS in } \\
\text { relapse }\end{array}$ & $\begin{array}{l}\text { SRNS in } \\
\text { remission }\end{array}$ & $\begin{array}{l}\text { SRNS in } \\
\text { relapse }\end{array}$ & $\begin{array}{c}P \\
\text { value }\end{array}$ \\
\hline Age (years) & $9(2-15)$ & $8(5-14)$ & $7(3-16)$ & $8(5-14)$ & .9 \\
\hline Age at onset (years) & $3.5(2.5-5)$ & $5(3-6)$ & $4(2-8)$ & $4.8(3-6)$ & .3 \\
\hline Height $(\mathrm{cm})$ & $123(80-165)$ & $128(94-154)$ & $115(85-152)$ & $118(95-152)$ & .6 \\
\hline Weight (kg) & $24(11-60)$ & $26(16-50)$ & $18(13-45)$ & $20(15-49)$ & .9 \\
\hline BMI & $17.6(13.2-23.8)$ & $17(15-21)$ & $16.5(13.6-19.5)$ & $\begin{array}{c}16.6(14.4- \\
23.9)\end{array}$ & .6 \\
\hline $\mathrm{SBP}(\mathrm{mmHg})$ & $110(90-120)$ & $110(90-120)$ & $110(100-120)$ & $110(100-120)$ & .4 \\
\hline $\mathrm{DBP}(\mathrm{mmH})$ & $70(60-90)$ & $70(60-80)$ & $70(60-80)$ & $70(60-80)$ & .8 \\
\hline Urea (mg/dl) & $20(12-40)$ & $23(17-29)$ & $21(14-45)$ & $30(21=80)$ & .02 \\
\hline Creatinine ( $\mathrm{mg} / \mathrm{dl})$ & $.4(.3-.8)$ & $.4(.4-.7)$ & $.4(.3-.9)$ & $.4(.3-.7)$ & .8 \\
\hline Albumin (gm/dl) & $4.3(3.7-4.7)$ & $1.9(1.2-2.5)$ & $3.7(3.3-4.6)$ & $1.9(1.6-2.6)$ & $<.001$ \\
\hline Cholesterol (mg/dl) & $142(84-190)$ & $309(249-336)$ & $178(115-322)$ & $280(225-600)$ & $<.001$ \\
\hline uRCR (mg/mg screat) & $.1(.01-.16)$ & $2.1(1.3-9.1)$ & $.1(.05-17)$ & $5.2(2-7)$ & $<.001$ \\
\hline Serum CXCL8/LL8 (pg/dI) & $59.6(1.6-296)$ & $114(5.8-296)$ & $73(13-276)$ & $293(11-296)$ & .19 \\
\hline $\begin{array}{l}\text { Urinary CXCL8/L8 (mg/ } \\
\text { mg ceart }\end{array}$ & $2.4(.0-9.8)$ & $40.6(24.4-80)$ & $38(21-57)$ & $187(61-210)$ & $<.001$ \\
\hline
\end{tabular}

Values are expressed as median (IQR)

$\mathrm{BMI}=$ body mass index, $\mathrm{SBP}=$ systolic blood pressure, $\mathrm{DBP}=$ diastolic blood pressure., $\mathrm{uPCR}=$ urine protein/creatinine, $\mathrm{CXCL8/ \textrm {IL } 8}$ =interleukin 8 
Table 2: Comparison between SRNS and SSNS groups during proteinurea

\begin{tabular}{|l|c|c|c|}
\hline & SSNS in relapse & SRNS in relapse & P value \\
\hline Albumin (gm/dl) & $1.9(1-2-2.5)$ & $1.9(1.6-2.6)$ & .9 \\
\hline Cholesterol (mg/dl) & $309(249-336)$ & $280(225-600)$ & .04 \\
\hline uPCR (mg/mg creat) & $2.1(1.3-9)$ & $5.2(2-7)$ & .35 \\
\hline Serum CXCL8/IL8 (pg/dl) & $114.3(5.8-296)$ & $293(11-296)$ & .04 \\
\hline Urinary CXCL8/IL8 (mg/ mg creat) & $40.6(24.4-80)$ & $187(61.8-210.3)$ & .04 \\
\hline
\end{tabular}

Values are expressed as median (IQR)

$\mathrm{uPCR}=$ urine protein/creatinine, CXCL8/IL8 =interleukin 8

Table 3: Comparison between SRNS and SSNS groups during remission

\begin{tabular}{|l|c|c|c|}
\hline & SSNS in remission & SRNS in remission & P value \\
\hline Albumin (gm/dl) & $4.3(3.7-4.7)$ & $3.7(3.3-4.6)$ & .9 \\
\hline Cholesterol (mg/dl) & $142(84-190)$ & $178(115-322)$ & .3 \\
\hline UPCR (mg/mg creat) & $.1(.01-.23)$ & $.1(.05-.17)$ & .9 \\
\hline Serum CXCL8/IL8 (pg/dl) & $59.6(1.6-296)$ & $73(13-276.8)$ & .9 \\
\hline Urinary CXCL8/IL8 (mg/ mg creat) & $2.4(.0-9.8)$ & $38(21.8-57)$ & .03 \\
\hline
\end{tabular}

Values are expressed as median (IQR)

$\mathrm{uPCR}=$ urine protein/creatinine, CXCL8/IL8 =interleukin 8

Table 4: Binary logistic regression test to study the association between steroid resistance and CXCL8/IL8

\begin{tabular}{|c|c|c|c|c|c|c|c|c|}
\hline & \multirow{2}{*}{ B } & \multirow{2}{*}{ S.E. } & \multirow{2}{*}{ Wald } & \multirow{2}{*}{$\mathrm{df}$} & \multirow{2}{*}{ Sig. } & \multirow{2}{*}{$\operatorname{Exp}(B)$} & \multicolumn{2}{|c|}{ 95\% C.I.for EXP(B) } \\
\hline & & & & & & & Lower & Upper \\
\hline Serum IL8 & .000 & .005 & .004 & 1 & .950 & 1.000 & .991 & 1.010 \\
\hline Urinary IL8 & .062 & .022 & 7.801 & 1 & .005 & 1.064 & 1.019 & 1.112 \\
\hline
\end{tabular}

The dependent variable is steroids resistance. Urinary CXCL8/IL8 is a good predictor of steroids resistance $(\mathrm{p}=.005)$ 


\section{IL8}

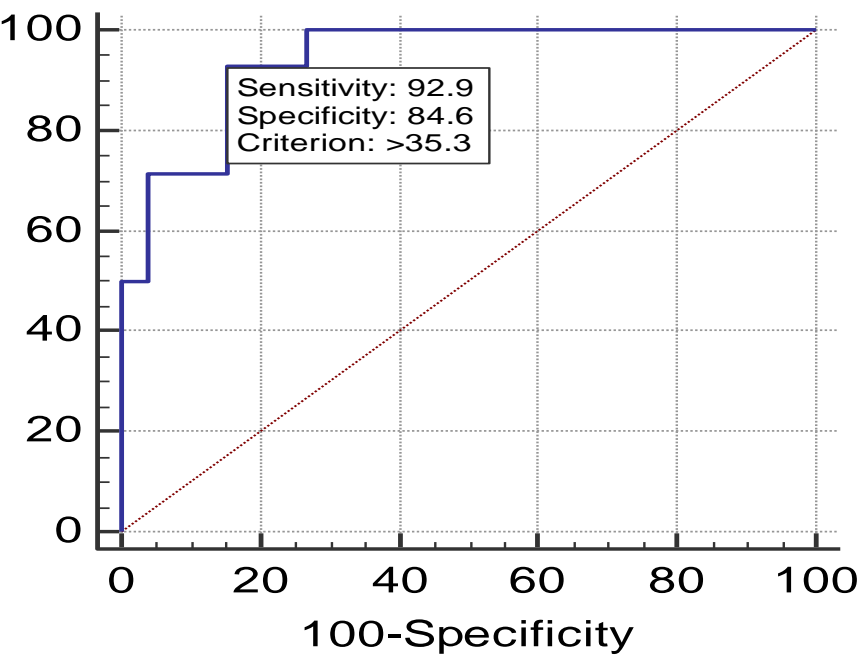

Figure1: Cutoff value for urine interleukin 8 (IL 8) levels above which steroid resistance is expected.

\section{Discussion}

The role of the immune factors in the pathogenesis of nephrotic syndrome has been discussed during the last few years. The activation of T-cells leads to release of cytokines that increase the glomerular permeability and provoke podocytes' barrier dysfunction with subsequent proteinuria.[10] . CXCL8/IL8 (CXCL8) is a chemokine produced by many cells, including macrophages, fibroblasts, renal mesangial, tubular cells and epithelial cells. [11]. Previous studies had reported the relation between CXCL8/IL8 and disease activity and linked high urinary CXCL8/IL8 levels to proteinurea $[6,13]$. We aimed in this study to clarify the relation between serum and urinary CXCL8/IL8 levels and responsiveness to steroid therapy. We found that during proteinuria both serum and urinary serum CXCL8/IL8 levels were significantly higher in SRNS than SSNS. On the other hand, serum CXCL8/IL8 levels were statistically insignificant between the SSNS and SRNS groups during remission while urinary CXCL8/IL8 levels were significantly higher in SRNS than SSNS in absence of statistically significant difference in urinary protein levels. We also found that high urinary IL8 levels is a predictor of steroid resistance as proved by logistic regression These findings may support that CXCL8/IL8 is produced by podocytes inducing an inflammatory process that is localized to the kidneys a finding that reported by Huber et al [14]. In their research, they studied the expression of chemocytokines receptors on podocytes and reported that podocytes can express receptors for CXCL8/IL8 and can release IL8 as well. Thus, these high levels of CXCL8/IL8 indicate severe podocyte injury with an ongoing inflammatory process. That could explain why some SRNS and frequent relapsing SSNS patient became dependent on immunosuppressive drugs and exhibit relapses on cessation of immune suppressive drugs [15]. In addition, the absence of significant difference between FSGS and MCD groups during disease activity again supports our hypothesis that increased urinary CXCL8/IL8 secretion is linked to the resistance to steroids and not to the underlying pathology. In contrast to findings reported by Lee et al, [16] who linked increased CXCL8/IL8 levels to MCD. The limitation of their study is that they involved only biopsy proven MCD patients and so the results were not compared with patients with other renal pathologies. CXCL8/IL8 was shown to induce changes in the permeability of the GBM via decreasing the synthesis of heparan sulfate proteoglycans, which provoke proteinuria in rats [17] . In human GN, the expression of CXCL8/IL8 has mainly been detected in association with glomerular cell proliferation and confined either to the expanded mesangial areas and crescents $[9,18,19]$ or spread over the whole glomeruli [19]. Podocytes express chemokines receptors and usually has the potential to respond to various soluble products of the immune system [20]. In our study, a cutoff value of $>35.3 \mathrm{pg} / \mathrm{mg}$ creatinine can be used to predict steroid resistance with $95 \%$ confidence interval of 0.81 to 0.99 corresponding to $93 \%$ sensitivity and $85 \%$ specificity and AUC of 0.94 . These findings strongly support that CXCL8/IL8 can be used as a biomarker for steroid responsiveness in INS.

\section{Conclusions}

Urinary CXCL8/IL8 levels were significantly higher in SRNS than SSNS patients during activity and remission with no significant difference on comparing MCD and FSGS patients. According to our findings, urinary CXCL8/IL8 can be used as a biomarker for steroid resistance in pediatric onset nephrotic syndrome.

Proposed possible application: early prediction of the response to steroid therapy in INS saving time and avoiding unnecessary medications. 


\section{References}

1. Bagga A: "Management of Steroid Sensitive Nephrotic Syndrome: Revised Guidelines".

2. Indian Pediatrics. 2008; 45:203-214

3. Gbadegesin R and Smoyer WE: "Nephrotic Syndrome". In: Comprehensive Pediatric Nephrology, 1 st edition, Geary DF, Schaefer F. (Eds): Mosby Elsevier, Philadelphia; 2008: 205-218.

4. International Study of Kidney Disease in Children. Nephrotic syndrome in children: prediction of histopathology from clinical and laboratory characteristics at time of diagnosis. A report of the International Study of Kidney Disease in Children. Kidney Int.1978; 13: 159-165

5. International Study of Kidney Disease in Children. The primary nephrotic syndrome in children. Identification of patients with minimal change nephrotic syndrome from initial response to prednisone. A report of the International Study of Kidney Disease in Children.

J Pediatr .1981;98:561-564

6. Camici M. The nephrotic syndrome is an immunoinflammatory disorder. MedHypotheses.2007; 68:900-905

7. Garin EH. Circulating mediators of proteinuria in idiopathic minimal lesion nephrotic syndrome. Pediatr Nephrol.2000; 14:872-878

8. van den Berg JG, Weening JJ . Role of the immune system in the pathogenesis of idiopathic nephrotic syndrome. Clin Sci (Lond).2004; 107:125-136

9. Bickel M. The role of interleukin-8 in inflammation and mechanisms of regulation. J Periodontol. 1993 May;64(5 Suppl):456-60

10. . Garin EH, West L, Zheng W. Effect of interleukin-8 on glomerular sulfated compounds and albuminuria. Pediatr Nephrol 1997; 11: 274-9.

11. Shimada M, Araya C, Rivard C, Ishimoto T, Johnson RJ, Garin EH. Minimal change disease: a "two-hit" podocyte immune disorder? Pediatr Nephrol. 2011; 26(4):645-649.
12. Pereira Wde F, Brito-Melo GE, Guimarães FT, Carvalho TG, Mateo EC, Simões e Silva AC. The role of the immune system in idiopathic nephrotic syndrome: a review of clinical and experimental studies. Inflamm Res. 2014; 63(1):1-12.

13. Vivarelli M, Massella L, Ruggiero B, Emma F. Minimal Change Disease. Clin J Am Soc Nephrol. 2017; 12(2):332-345.

14. Souto MF, Teixeira AN, Russo RC, Penido MG, Silveira KD, Teixeira MM, and Silva AS. Immune Mediators in Idiopathic Nephrotic Syndrome: Evidence for a Relation between Interleukin 8 and Proteinuria. Pediatric Research .2008; 64(6).

15. Huber T B, Reinhardt HC, Exner M, Burger J A, Kerjaschki, D, Saleem, MA, Pavenstädt, H. Expression of Functional CCR and CXCR Chemokine Receptors in Podocytes. J Immunol 2002; 168:6244-6252.

16. Durkan A. M., Hodson E. M., Willis N. S., Craig J. C. Immunosuppressive agents in childhood nephrotic syndrome: a meta-analysis of randomized controlled trials. Kidney Int. 2001 May; 59(5): 1919-1927.

17. Lee H, S, Choe B, H, Kwon S, H, Chung K, Y, Koo J, $\mathrm{H}, \mathrm{Ko} \mathrm{C}, \mathrm{W}$, Interleukin-8 and Tumor Necrosis FactorAlpha Are Increased in Minimal Change Disease but Do Not Alter Albumin Permeability. Am J Nephrol . 2003;23:260-266

18. Pan Q, Wu J, Tao J, et al. Role of basophils in the pathogenesis of minimal change nephrotic syndrome: a literature review. Exp Ther Med. 2014;8(4):1027-1031

19. Lim CS, Yoon HJ, Kim YS, Ahn C, Han JS, Kim S, Lee JS, Lee HS, Chae DW: Clinicopathological correlation of intrarenal cytokines and chemokines in IgA nephropathy. Nephrology. 2003, 8, 21-27.

20. Cockwell P, Brooks CJ, Adu D, Savage COS: Interleukin-8: a pathogenetic role in antineutrophil autoantibody associated glomerulonephritis. Kidney Int 1999;55:852-863

21. Wada T, Tomosugi N, Naito T, Yokoyama H, Kobayashi K, Harada A, Mukaida N, Matsushima K: Prevention of proteinuria by the administration of anti-interleukin 8 antibody in experimental acute immune complex-induced glomerulonephritis. J Exp Med 1994, 180, 1135-1140.

\section{Declaration \\ Statement}

Ethics approval and consent to participate

The study was permitted by the local ethical committee and in accordance with Helsinki declaration of Bioethics and its later amendments. Informed consent was obtained from all participants caregivers.

Funding

The authors declare that they didn't receive any financial support from agencies or others

Competing interests

No conflicts of interest to be declared by the authors.

Acknowledgements Not applicable. 